\title{
Screening for fetal aneuploidy and neural tube defects
}

\author{
Deborah A. Driscoll, $M D^{l}$, and Susan J. Gross, $M D^{2}$; for the Professional Practice Guidelines Committee
}

\begin{abstract}
Disclaimer: This guideline is designed primarily as an educational resource for medical geneticists and other health care providers to help them provide quality medical genetic services. Adherence to this guideline does not necessarily assure a successful medical outcome. This guideline should not be considered inclusive of all proper procedures and tests or exclusive of other procedures and tests that are reasonably directed to obtaining the same results. In determining the propriety of any specific procedure or test, the geneticist should apply his or her own professional judgment to the specific clinical circumstances presented by the individual patient or specimen. It may be prudent, however, to document in the patient's record the rationale for any significant deviation from this guideline.
\end{abstract}

\begin{abstract}
Maternal serum screening for neural tube defects and fetal aneuploidy in the second trimester has been incorporated into obstetrical practice over the past two decades. Now, as a result of several multicenter trials, first trimester screening between 11 and 14 weeks has been shown to be an effective and reliable screening test for Down syndrome and trisomy 18. This policy updates the American College of Medical Genetics policy statement entitled Second Trimester Maternal Serum Screening for Fetal Open Neural Tube Defects and Aneuploidy (2004), incorporates First trimester diagnosis and screening for fetal aneuploidy (2008) and complements the sections of American College of Medical Genetic's Standards and Guidelines for Clinical Genetics Laboratories entitled Prenatal Screening for Down syndrome (2005) and Prenatal Screening for Open Neural Tube Defects (2005). Genet Med 2009:11(11):818-821.
\end{abstract}

Key Words: aneuploidy, multiple marker screening, nuchal translucency

S creening tests to identify women at risk for fetal aneuploidy

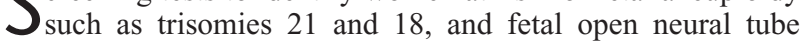
defects (ONTDs) are routinely offered to all women during pregnancy regardless of maternal age. A number of screening strategies are available, which utilize both biochemical and ultrasonography to achieve high detection rates. Maternal serum screening in the second trimester has now been available for over two decades. More recently, first trimester screening tests offer women the opportunity of early screening for fetal aneuploidy and the option of earlier diagnosis. Further, maternal age 35 is no longer recommended as a cutoff for determining which patients should be offered invasive prenatal diagnostic testing. ${ }^{1}$ This current guideline supercedes the American College of

From the ${ }^{1}$ University of Pennsylvania Medical Center, Philadelphia, Pennsylvania; and ${ }^{2}$ Department of Obstetrics and Gynecology, North Bronx Healthcare Network, Albert Einstein College of Medicine of Yeshiva University, Bronx, New York.

Deborah A. Driscoll, MD, Department of Obstetrics/Gynecology, 3400 Spruce St, 5 Dulles Building, Philadelphia, PA 19104. E-mail: ddriscoll@ obgyn.upenn.edu.

Disclosure: Dr. Driscoll declares no conflicts of interest. For Dr. Gross's disclosure, see Acknowledgments section at the end of the article.

Submitted for publication August 3, 2009

Accepted for publication August 6, 2009.

DOI: $10.1097 /$ GIM.0b013e3181bb267b
Medical Genetics (ACMG) policy statements entitled Second Trimester Maternal Serum Screening for Fetal ONTDs and Aneuploidy (2004) and First Trimester Diagnosis and Screening for Fetal Aneuploidy (2008) and complements the sections of ACMG's Standards and Guidelines for Clinical Genetics Laboratories entitled Prenatal Screening for ONTDs and Prenatal Screening for Down syndrome (DS) (http://www.acmg.net).

\section{SCREENING FOR NEURAL TUBE DEFECTS}

In the 1980s, maternal serum screening programs became available to identify pregnancies at risk for ONTDs and anencephaly; $75-90 \%$ of ONTDs and $\geq 95 \%$ of anencephaly can be detected by elevated maternal serum alphafetoprotein (MSAFP) levels with a screen positive rate of $5 \%$ or less. ${ }^{2}$ MSAFP screening may also detect $85 \%$ of ventral wall defects. ${ }^{3}$

The optimal time for NTD screening is 16-18 weeks' gestation, but screening can be done between 15 and 20 weeks. Although NTD screening is optional, pregnancy management is altered by the identification of a fetal neural tube defect independent of the patient's decision to carry or discontinue her pregnancy. The laboratory should be informed of the gestational age at the time the sample was drawn for an accurate interpretation. If there is a discrepancy in gestational age of $>10$ days after an ultrasound examination, then the test result must be reinterpreted. ${ }^{4}$ If the sample was drawn at $<15$ weeks, then a new sample should be sent with the accurate gestational age. Ultrasound dating of the pregnancy reduces the false-positive rate and increases the detection rate of ONTDs. The test is most accurate if the laboratory is also informed of maternal weight, race (white or Black/African American), presence of insulin dependent diabetes, number of fetuses, and family history of ONTD. These factors are used to adjust the multiples of the median (MoM) level. Cutoff levels for ONTD screening are 2.0-2.5 MoM in singleton pregnancies and 4.0-5.0 MoM in twin pregnancies. ${ }^{4}$ However, the specific cutoff value is laboratory dependent, and thus screen positive and screen negative results are specified on reports. Genetic counseling and additional testing such as targeted ultrasound examination and/or amniocentesis to evaluate amniotic fluid AFP, and acetylcholinesterase levels are recommended for pregnancies with an elevated MSAFP test result. 5 


\section{DIAGNOSIS OF FETAL ANEUPLOIDY}

Historically, women 35 years or older at the time of delivery and women with an abnormal screening test were offered prenatal genetic counseling and the option of a diagnostic test such as amniocentesis or chorionic villus sampling (CVS). CVS is performed in the first trimester from 10 through 13 weeks' gestation, whereas amniocentesis can be performed starting at 15 weeks' gestation. CVS can be a transcervical or transabdominal procedure. The risk of pregnancy loss with CVS is low; some reports suggest this risk may be lower for transabdominal procedures $^{6}$; however, other reports suggest an equal loss rate between the two procedures. ${ }^{7,8}$ Differences in loss rates if any likely reflect operator experience with transcervical procedures and available ultrasound technology. Contemporary literature suggests that the risk of pregnancy loss from sonographically directed amniocentesis is 1 in $300-600$, with one study suggesting that there may be no significant increase over the background miscarriage risk. ${ }^{8,9}$ Based on the relative safety of these diagnostic procedures, the inability of screening tests to detect all types of chromosomal abnormalities and in deference to patient preference and autonomy, the American College of Obstetricians and Gynecologists recommends that all pregnant women, regardless of maternal age, should have the option of invasive diagnostic testing. Diagnostic testing should be made available, if requested, after appropriate counseling including risks and benefits. ${ }^{1,8}$

Maternal age alone is a poor screening criterion and therefore, for women who would like to further refine their risk before deciding whether or not to undergo invasive testing, screening provides such an option. The current screening tests used to adjust a woman's age risk for trisomies 21 and 18 have been shown to be highly effective and useful in several large, prospective multicenter studies. ${ }^{10-14}$ These screening tests are voluntary and, hence, women who do not want any information regarding the chromosomal status of their fetus should not be required to undergo testing or screening.

\section{FIRST TRIMESTER SCREENING FOR FETAL ANEUPLOIDY}

The most effective screening test in the first trimester uses a combination of biochemical markers, pregnancy-associated plasma protein A (PAPP-A) and human chorionic gonadotropin (hCG), and the nuchal translucency (NT) measurement to adjust a woman's risk for trisomies 21 and 18. Collection of blood for biochemical analysis is performed between 9 and 13 6/7 weeks' gestation (crown rump length, 24-84 mm). ${ }^{15-17}$ Ultrasound assessment of the NT measurement is performed between 11 and 13 6/7 weeks (crown rump length, 45-84 $\mathrm{mm}$ ). In four major studies, the DS detection rates range from 79 to $90 \%$ with a $5 \%$ false-positive screen rate. ${ }^{11-14}$ Typically, PAPP-A levels are reduced, and hCG is increased in trisomy 21 . The overall data suggest that free $\beta$-hCG has slightly increased sensitivity when compared with intact hCG. ${ }^{15}$ However, access to free $\beta$-hCG has been limited and therefore, many programs in the United States use intact hCG. Free $\beta$-hCG and intact hCG levels are reduced in trisomy 18 . Women who have first trimester screening and/or CVS should be offered MSAFP screening between 15 and 20 weeks' gestation and/or an ultrasound examination for the detection of NTDs between 15 and 20 weeks' gestation.

NT refers to a clearly demarcated fluid filled or sonolucent space behind the fetal neck. This space is present in all fetuses. An increased NT measurement is significantly associated with trisomy 21 and other forms of aneuploidy. ${ }^{16,17}$ NT measurement alone has a detection rate for DS of $\sim 70 \%$ with a $5 \%$ falsepositive rate. In general, NT measurement has limited usefulness as a stand-alone test because of the increased sensitivity achieved with incorporation of first-trimester analytes or serum markers. ${ }^{1}$ Under some circumstances such as a NT measurement of $4 \mathrm{~mm}$ or greater or a cystic hygroma where the addition of serum markers in the first trimester does not significantly change the risk for DS, and it is reasonable to offer the patient a diagnostic test. ${ }^{18,19}$

Combined first trimester serum screening for multifetal gestations is less sensitive $(70 \%)$ than in singleton pregnancies, and there are concerns about averaging serum markers. NT measurement alone yields similar detection rates for DS in multiple gestations. However, an increased or discordant NT may signify an adverse pregnancy outcome particularly in monochorionic twins. ${ }^{20}$

Increased NT measurements are also associated with other fetal structural anomalies including congenital heart defects, diaphragmatic hernias, skeletal dysplasias, and a variety of genetic syndromes. Based on the current scientific evidence American College of Obstetricians and Gynecologists recommends that patients with a fetal NT measurement of $3.5 \mathrm{~mm}$ or higher should be offered a targeted ultrasound, echocardiogram, or both. ${ }^{1}$

\section{SECOND TRIMESTER SCREENING FOR FETAL ANEUPOLOIDY}

Multiple marker screening is used in the second trimester (15-20 weeks) to screen for trisomies 21 and 18 as well as ONTDs. The most widely used second trimester screening test is the "quad screen," so named because it uses four biochemical markers - alphafetoprotein (AFP), hCG, unconjugated estriol (uE3), and dimeric inhibin-A. Using a second trimester DS cutoff risk of 1 in 270, the combination of maternal age and the "quad screen" detects $\sim 75 \%$ of DS cases in women who are younger than 35 years with a positive screening rate of $5 \%$ and $>80 \%$ of the DS fetuses in women 35 years and older. ${ }^{21}$ In most cases of DS, the AFP and $\mathrm{uE} 3$ levels are lower, whereas hCG and dimeric inhibin-A levels are higher. The "triple test" that relies on only three of the four analytes (AFP, hCG, and uE3) has a sensitivity of $\sim 65 \%$ for DS and $70 \%$ for trisomy 18 . In most cases of trisomy 18 , the levels of all three analytes are low. Maternal serum screening in the second trimester has been validated and is an appropriate screening test for both DS and trisomy 18. In twin gestations, second trimester serum screening detects only $\sim 50 \%$ of affected fetuses and can be difficult to interpret because a normal twin may mask the results of an affected fetus. ${ }^{22}$ There are insufficient prospective studies to determine actual detection rates in a given program; thus, laboratories that provide risk results are using mathematical models.

Multiple marker screening in the second trimester does not reliably detect the other forms of aneuploidy that occur more often as result of increasing maternal age, such as trisomy 13 and Klinefelter syndrome (47,XXY) ( 1 in 20,000 and 1 in 1000 livebirths, respectively). These would be detected on a routine cytogenetic analysis of cultured amniocytes or villi.

Multiple marker screening can be performed at 15-20 weeks' gestation. The laboratory must be informed of the gestational age at the time the sample was drawn for an accurate interpretation. The first day of the last menstrual period is the most common method for assessing gestational age, but ultrasound measurement of the crown-rump length in the first-trimester or a biparietal diameter in the second trimester provides a more accurate estimate of gestational age, within 7 and 10 days, 
respectively. If the gestational age changes by $>10$ days after an ultrasound examination, then the test results must be reinterpreted. If the sample was drawn at $<15$ weeks, a new sample should be obtained and analyzed in accordance with the corrected gestational age. ${ }^{23}$

Second trimester ultrasonography may identify fetal anatomic defects, such as congenital heart defect or markers suggestive of fetal aneuploidy like a thickened nuchal fold, absent nasal bone, renal pyelectasis, or echogenic bowel. However, these sonographic makers have a low sensitivity and specificity for detecting aneuploidy; therefore, ultrasonography is not recommended as a primary screening tool for fetal aneuploidy. ${ }^{24,25}$

\section{FIRST AND SECOND TRIMESTER SCREENING FOR ANEUPLOIDY}

There have been several different approaches proposed to improve screening detection rates, which take advantage of both first and second trimester screening tests. Integrated screening provides a single revised risk assessment based on the NT measurement and PAPP-A in the first trimester and the second trimester quad screen. Studies have shown that this approach has the highest detection rate for trisomy $21(93-96 \%)^{11,13}$; however, first trimester results must be withheld until the test is completed and, hence, a patient does not have the option of an early diagnostic test in the event that the first trimester results would have indicated an increased risk for aneuploidy. Serum integrated screening provides the patient with a single ageadjusted risk assessment for trisomies 18 and 21 based on a first trimester PAPP-A and the second trimester serum screen. This approach has a detection rate of $\sim 88 \%$ and is useful when a NT measurement cannot be obtained, or in programs or communities where NT measurement is unavailable. ${ }^{26}$

Two sequential screening approaches, stepwise and contingency, have been introduced to allow disclosure of results in the first trimester. Both have high detection rates $(\sim 90 \%)$ with low positive screening rates of 2-3\%. ${ }^{27-29}$ By disclosing first trimester results in stepwise screening, a patient at high risk can undergo a CVS and earlier termination in the event of an affected fetus. A patient with a low or moderate risk after the first trimester screen proceeds to second trimester screening and receives a final revised risk assessment based on first and second trimester results. With contingency screening only those women with a first trimester screening result in a laboratory determined intermediate range undergo testing in the second trimester. Independent assessment of DS risk by first and second trimesters screening is discouraged because of the high falsepositive rates associated with this approach. ${ }^{11}$ It should be emphasized that stated improved detection rates for all combined methodologies discussed above are based on mathematical modeling techniques for combining first and second trimester results, not large prospective cohort studies such as are available for first trimester alone or second trimester alone. A disadvantage of the serum integrated or the sequential screening approach is the real possibility that the patient may not return for the second blood sample..$^{30}$

\section{RECOMMENDATIONS}

The ACMG recommends the following.

- All women should have the option of invasive diagnostic testing for fetal aneuploidy by CVS, if available, or amniocentesis. Benefits include diagnosis of all major aneuploidies and large chromosomal rearrangements.
- For women who do not initially want a diagnostic test, screening for aneuploidy and ONTDs should be offered to those who present for prenatal care before 20 weeks' gestation regardless of maternal age. In cases where women do not want information regarding fetal aneuploidy status, the reason for declining screening should be documented.

- First trimester screening (NT measurement, PAPP-A, and hCG) is an acceptable, effective approach for screening for fetal aneuploidy if a woman presents early in pregnancy (before 14 weeks' gestation).

- Providers and/or centers that use aneuploidy screening approaches based on a combination of first and second trimesters screening (integrated, serum integrated, and sequential) must be prepared to ensure that there is adequate follow-up. If integrated screening is offered, they should be certain that nondisclosure is acceptable to the patient. Sequential or contingency screening with disclosure of first trimester results may be preferable for some providers and patients.

- Women should be informed of the adjusted risk for aneuploidy and allowed to make individual decisions regarding diagnostic testing based on the numerical risk. Individuals will weigh risk/benefits surrounding diagnostic testing differently.

- Women who decide to undergo first trimester screening and/or CVS should be offered MSAFP screening and/or an ultrasound for the detection of neural tube defects between 15 and 20 weeks' gestation.

- First trimester screening or second trimester screening can be used in multifetal pregnancies; however, women should be made aware of the limitations of screening in this setting.

- A NT measurement of $3.5 \mathrm{~mm}$ or greater, even in the setting of a low risk for aneuploidy based on screening results or a normal fetal karyotype, should alone prompt a detailed anatomic ultrasound, echocardiogram or both.

\section{ACKNOWLEDGMENTS}

Dr. Gross reports receiving grant support from PerkinElmer and Genzyme and being the inventor on a patent filed by the Albert Einstein School of Medicine on the use of cytokeratins as a potential serum marker for aneuploidy, for which she has received no royalties. Dr. Gross has served as a consultant for Luminex Molecular Diagnostics Inc. and is participating in a research collaboration to validate Jewish Genetics screening assays.

\section{REFERENCES}

1. ACOG Committee on Practice Bulletins. ACOG Practice Bulletin Number 77: screening for fetal chromosomal abnormalities. Obstet Gynecol 2007; 109:217-227.

2. Wald NJ, Cuckle HS, Brock JH, Peto R, Polani PE, Woodford FP. Maternal serum alpha-fetoprotein measurement in antenatal screening for anencephaly and spina bifida in early pregnancy. Report of the U.K. Collaborative Study on alpha-etoprotein in relation to neural tube defects. Lancet 1977;1:1323-1332.

3. Milunsky A. Maternal serum screening for neural tube and other defects. In: Milunsky A, editor. Genetic disorders and the fetus: diagnosis, prevention, and treatment. 4th ed. Baltimore, MD: Johns Hopkins University Press, 1998:635-701.

4. Bradley LA, Palomaki GE, McDowell GA; ONTD Working Group; ACMG Laboratory Quality Assurance Committee. Technical standards and guidelines: prenatal screening for open neural tube defects. Genet Med 2005;7: 355-369.

5. Cheschier N. ACOG Committee on Practice Bulletins-Obstetrics. ACOG Practice Bulletin Number 44: neural tube defects. Int J Gynaecol 2003;83: 123-133.

6. Alfirevic Z, Sundberg K, Brigham S. Amniocentesis and chorionic villus sampling for prenatal diagnosis. Cochrane Database Syst Rev 2003;3: CD003252. 
7. Jackson LG, Zachary JM, Fowler SE, et al. The US National Institute of Child Health and Human Development Chorionic-Villous Sampling and Amniocentesis Study Group. A randomized comparison of transcervical and transabdominal chorionic-villous sampling. N Engl J Med 1992;327:594598.

8. ACOG Committee on Practice Bulletins. ACOG Practice bulletin Number 88: invasive prenatal testing for aneuploidy. Obstet Gynecol 2007;110:1459-1466.

9. Eddelman KA, Malone FD, Sullivan L, et al. Pregnancy loss rates after midtrimester amniocentesis. Obstet Gynecol 2006;108:1067-1072.

10. Kagan KO, Wright D, Baker A, Sahota D, Nicolaides KH. Screening for trisomy 21 by maternal age, fetal nuchal translucency thickness, free betahuman chorionic gonadotropin and pregnancy-associated plasma protein-A. Ultrasound Obstet Gynecol 2008;31:618-624.

11. Malone F, Canick JA, Ball RH, et al. First- and Second-Trimester Evaluation of Risk (FASTER) Research Consortium. First-trimester or second-trimester screening, or both, for Down's syndrome. N Engl J Med 2005;353:2001-2011.

12. Wapner R, Thom E, Simpson JL, et al. First Trimester Maternal Serum Biochemistry and Fetal Nuchal Translucency Screening (BUN) Study Group. First-trimester screening for trisomies 21 and 18. $N$ Engl J Med 2003;349:1405-1413

13. Wald NJ, Rodeck C, Hackshaw AK, Walters J, Chitty L, Mackinson AM; SURUSS Research Group. First and second trimester antenatal screening for Down's syndrome: the results of the Serum Urine and Ultrasound Screening Study (SURUSS) [erratum appears in J Med Screen 2006;13:51-52]. Health Technol Assess 2003;7:1-77.

14. Spencer K, Spencer CE, Power M, Dawson C, Nicolaides KH. Screening for chromosomal abnormalities in the first trimester using ultrasound and maternal serum biochemistry in a one-stop clinic: a review of three years prospective experience. BJOG 2003;110:281-286.

15. Evans MI, Krantz DA, Hallahan TW, Galen RS. Meta-analysis of first trimester Down syndrome screening studies: free beta-human chorionic gonadotropin significantly outperforms intact human chorionic gonadotropin in a multimarker protocol. Am J Obstet Gynecol 2007;196:198-205.

16. Nicolaides KH. Nuchal translucency and other first-trimester sonographic markers of chromosomal abnormalities. Am J Obstet Gynecol 2004;191:45-67.

17. Snijders RJ, Noble P, Sebire N, Souka A, et al. Fetal Medicine Foundation First Trimester Screening Group. UK multicenter project on assessment of risk of trisomy 21 by maternal age and fetal nuchal-translucency thickness at 10-14 weeks of gestation. Lancet 1998;351:343-346.
18. Comstock $\mathrm{CH}$, Malone FD, Ball RH, et al. FASTER Research Consortium. Is there a nuchal translucency millimeter measurement above which there is no added benefit from first trimester screening? Am J Obstet Gynecol 2006; $195: 843-847$.

19. Malone FD, Ball RH, Nyberg DA, et al. FASTER Trial Research Consortium. First-trimester septated cystic hygroma; prevalence, natural history, and pediatric outcome. Obstet Gynecol 2005;106:288-294.

20. Cleary-Goldman J, Berkowitz RL. First trimester screening for Down syndrome in multiple pregnancies. Semin Perinatol 2005;29:395-400.

21. Benn PA, Fang M, Egan JF, Horne D, Collins R. Incorporation of inhibin-A in second trimester screening for Down syndrome. Obstet Gynecol 2003; 101:451-454.

22. Neveux LM, Palomaki G, Knight GJ, Haddow JE. Multiple marker screening for Down syndrome in twin pregnancies. Prenat Diagn 1996;16:29-34.

23. Palomaki GE, Bradley LA, McDowell GA; Down Syndrome Working Group; ACMG Laboratory Quality Assurance Committee. Technical standards and guidelines: prenatal screening for Down syndrome. Genet Med 2005; $7: 344-354$

24. Smith-Bindman R, Chu P, Goldberg JD. Second trimester prenatal ultrasound for the detection of pregnancies at increased risk for Down syndrome. Prenat Diagn 2007;27:535-544.

25. ACOG Committee on Practice Bulletins. ACOG Practice Bulletin Number 101: ultrasonography in pregnancy. Obstet Gynecol 2009;113:451-461.

26. Knight GJ, Palomaki GE, Neveux LM, et al. Integrated serum screening for Down syndrome in primary obstetric practice. Prenat Diagn 2005;25:1162-1167.

27. Platt LD, Greene N, Johnson A, et al; First Trimester Maternal Serum Biochemistry and Fetal Nuchal Translucency Screening (BUN) Study Group. Sequential pathways of testing after first-trimester screening for trisomy 21. Obstet Gynecol 2004;104:661-666.

28. Wright D, Bradbury I, Benn P, Cuckle H, Ritchie K. Contingent screening for Down syndrome is an efficient alternative to non-disclosure sequential screening. Prenat Diagn 2004;24:762-766.

29. Palomaki GE, Steinort K, Knight G, Haddow JE. Comparing three screening strategies for combining first- and second-trimester Down syndrome markers. Obstet Gynecol 2006;107:367-375.

30. Weisz B, Pandya P, Chitty L, Jones P, Huttly W, Rodeck C. Practical issues drawn from the implementation of the integrated test for Down syndrome screening into routine clinical practice. BJOG 2007;114:493-497. 